\title{
Coding repeats coordinate repression
}

Most characterized binding sites for microRNAs (miRNAs) lie in the 3' UTRs of their target mRNAs. The few that have been found in ORFs or 5' UTRs are generally thought to confer less effective mRNA repression, perhaps because the translation machinery displaces the miRNA silencing complex from these sites. Now, a study has unexpectedly found clusters of ORF target sites that mediate substantial repression; this work also suggests a role for coding-region repeat sequences in coordinated gene regulation.

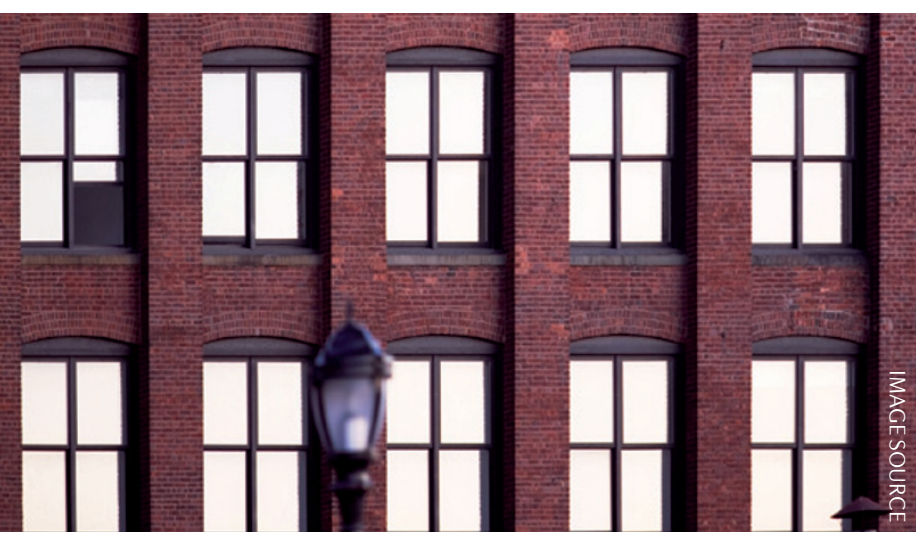

By looking at gene-expression data from human cells transfected with miR-181a, Schnall-Levin, Berger, Bartel and colleagues noticed strong downregulation of genes that contained several miR-181 sites in their coding regions. These sites were ' 8 -mers' - that is, sites where 8 nucleotides of the mRNA pair with the $5^{\prime}$ end of the miRNA (the 'seed'). These 8-mer sites generally elicit more efficient repression than 6-mer or 7-mer sites. It is unusual for an mRNA to have several sites for a single miRNA, but the authors found 52 genes with four or more miR-181 ORF 8-mer sites; luciferase reporter assays showed that the ORF sites are responsible for repression.

Next, the authors searched for any 8-mers that occur four or more times in at least 25 human ORFs. They found 3348 -mers that met these criteria, seven of which matched the seed sequences of known miRNA families. For four of these miRNA families, the predicted set of target mRNAs was almost exclusively composed of $\mathrm{C}_{2} \mathrm{H}_{2}$ zinc finger genes. These genes form the largest class of transcription factors and have tandemly repeated zinc finger motifs, which is where the miRNA sites lie. As with the miR-181 targets, Schnall-Levin et al. demonstrated that the ORF sites in these predicted target mRNAs lead to effective repression.

By phylogenetic analysis, the authors showed that repeated miRNA sites arose from intragenic duplications of the domain containing the site, and these were followed by multiple gene duplications. Therefore, the same miRNA can target a large group of related genes, giving the opportunity for coordinated regulation. More broadly, the authors suggest that coding-sequence repeats - of which there are many different types - could be involved in directing other post-transcriptional regulatory mechanisms.

\section{Mary Muers}

ORIGINAL RESEARCH PAPER Schnall-Levin, M. et al. Unusually effective microRNA targeting within repeat-rich coding regions of mammalian mRNAs. Genome Res. 17 Jun 2011 (doi:10.1101/ gr.121210.111)

FURTHER READING Ghildiyal, M. \& Zamore, P. D. Small silencing RNAs: an expanding universe.

Nature Rev. Genet. 10, 94-108 (2009) 